\title{
GENERATORS FOR THE BOUNDED AUTOMORPHISMS OF INFINITE-RANK FREE NILPOTENT GROUPS
}

\author{
R.G. Burns and Lian PI
}

\section{To B.H. Neumann on his eightieth birthday}

\begin{abstract}
It is shown that the natural generalisations of the elementary Nielsen transformations of a free group to the infinite-rank case, furnish generators for the subgroup of "bounded" automorphisms of any relatively free nilpotent group of infinite rank. This settles the nilpotent analogue of a question of $D$. Solitar concerning the "bounded" automorphisms of absolutely free groups of infinite rank.
\end{abstract}

\section{INTRODUCTION}

In the search for a simple set of generators of the automorphism group of a free group $F$ on an infinite set $\left\{x_{i} \mid i \in I\right\}$ of free generators, the following natural generalisations of the elementary Nielsen transformations (see for example [7]) suggest themselves:

(i) automorphisms permuting the $x_{i}$;

(ii) automorphisms inverting any subset of the $x_{i}$ 's and leaving the remainder fixed;

(iii) automorphisms of the following form: given any partition $I=I_{1} \amalg I_{2}$, set $x_{i_{1}} \mapsto x_{i_{1}} x_{i_{2}}$ for each $i_{1} \in I_{1}$ and any choice of $i_{2} \in I_{2}$; and $x_{i_{2}} \mapsto x_{i_{2}}$ for all $i_{2} \in I_{2}$. (Thus each $x_{i_{1}}$ is post-multiplied by some $x_{i_{2}}$, where $i_{2}$ may vary with $i_{1}$.)

It will be convenient to consider also automorphisms of the following type, though generated by those of types (ii) and (iii):

(iv) as in (iii) except that now pre- as well as post-multiplication is permitted, and by any $x_{i_{2}}^{-1}, i_{2} \in I_{2}$, as well as $x_{i_{2}}$; thus $x_{i_{1}} \mapsto x_{i_{1}} x_{i_{2}}^{ \pm 1}$ or $x_{i_{2}}^{ \pm 1} x_{i_{1}}$.

We call automorphisms of these four types generalised elementary Nielsen trans. formations (briefly, GENTs).

However, the automorphisms of types (i), (ii), (iii) (and so also (iv)) do not generate the full automorphisin group of $F$, since clearly any automorphism $\varphi$ which is a finite

Received 31 May 1989

The first author would like to thank members of the Department of Mathematics of the University of Queensland, for their hospitality.

Copyright Clearance Centre, Inc. Serial-fee code: 0004-9729/89 \$A2.00+0.00. 
product of these will be (freely) bounded in the sense that the lengths of the words $x_{i} \varphi$ and $x_{i} \varphi^{-1}$ are bounded: there is an $N$ such that $\left|x_{i} \varphi\right|,\left|x_{i} \varphi^{-1}\right| \leqslant N$ for all $i \in I$. Since the bounded automorphisms form a subgroup of Aut $F$, it is natural to ask the following

Question. (Solitar) Is the group of all automorphisms of $F$ that are bounded relative to the given basis $\left\{x_{i} \mid i \in I\right\}$, generated by the GENTs?

R. Cohen [4] has shown that if $\varphi$ is bounded by $N \leqslant 3$, then $\varphi$ is a product of finitely many GENTs. The question remaining unsettled for larger $N$, it becomes natural to consider its analogue for relatively free groups $F / V$ all of whose automorphisms are induced by automorphisms of $F$. This is known to be the case when $F / V$ is nilpotent (see [1]), and it is in this context that we give an affirmative answer.

'Io formulate our result a few preliminaries are needed. With $F$, as above, absolutely free with infinite free basis $\left\{x_{i} \mid i \in I\right\}$, let $V$ be a characteristic subgroup of $F$ containing $\gamma_{c+1}(F)$, the $(c+1)$ st term of the lower central series of $F \cdot(B y[2] V$ must then in fact be fully invariant in $F$.) It is well known (see for example [5]) that every element of $F / V$ may be written in the form

$$
\left(x_{i_{1}}^{m_{1}} x_{i_{2}}^{m_{2}} \ldots x_{i_{k}}^{m_{k}} c_{j_{1}}^{n_{1}} c_{j_{2}}^{n_{2}} \ldots c_{j_{l}}^{n_{l}}\right) V
$$

where $k, l \geqslant 0$, the $m$, are non-zero integers, the $n_{t}$ are positive integers, $i_{1}, i_{2}, \ldots, i_{k}$ are distinct elements of $I$, and the $c_{j_{t}}$ are distinct left-normed commutators in the $x_{i}$, whose weights satisfy wt $_{j_{1}} \leqslant$ wtc $_{j_{2}} \leqslant \ldots \leqslant$ wt $c_{j_{l}} \leqslant c$. We shall say that an automorphism $\theta$ of $F / V$ is (nilpotently) bounded if there is an integer $N=N(\theta)$ such that for every $i \in I$ there are expressions for $\left(x_{i} V\right) \theta$ and $\left(x_{i} V\right) \theta^{-1}$ of the form (1) satisfying

$$
\left|m_{1}\right|+\ldots+\left|m_{k}\right|+n_{1}+\ldots+n_{l} \leqslant N
$$

Our main result is as follows:

ThEOREM 1.1. Every bounded automorphism $\theta$ of $F / V$ is induced by an automorphism of $F$ which is a finite product of GENTs of type (iv).

We shall in the sequel use the word genetic to refer to automorphisms of $F$ expressible as finite products of GENTs of type (iv).

Remark 1. It is not difficult to see that every bounded automorphism of $F$ induces a bounded automorphism of $F / V$. On the olher hand by our theorem every bounded automorphism of $F^{\prime} / V$ lifts to a genetic automorphism of $F$. Hence we draw the following conclusion, which may be of interest in connection with Solitar's question: 
Corollary 1.2. Let $\varphi$ be any bounded automorphism of the infinite-rank free group $F$. Corresponding to each characteristic subgroup $V$ of $F$ such that $F / V$ is nilpotent, there are automorphisms $\psi_{V}$ and $\eta_{V}$ of $F$ such that $\psi_{V}$ is genetic, $\eta_{V}$ induces the identity automorphism of $F / V$, and $\varphi=\psi_{V} \eta_{V}$.

Remark 2. The case where $F / V$ is free abelian of countable rank has been established by Macedońska-Nosalska [6]. Our proof in the abelian situation (more general than in [6] since we allow finite exponent and make no countability requirement of $I$ ) is obtained by modifying mildly an argument of Swan appearing in [3].

Remark 3. Automorphisms of $F$ resembling those of type (iv) except that we allow $x_{i_{1}} \mapsto x_{i_{1}} x_{i_{2}}^{n}$ or $x_{i_{2}}^{n} x_{i_{1}}$, that is pre- or post-multiplication of the $x_{i_{1}}, i_{1} \in I_{1}$, by arbitrary powers of the $x_{i_{2}}$, are clearly unbounded precisely if the exponents $n$ are unbounded. A more complex, though standard, example of an unbounded automorphism of $F($ or $F / V$ ) is the following one:

$$
x_{1} \mapsto x_{1}, x_{2} \mapsto x_{1} x_{2}, x_{3} \mapsto x_{2} x_{3}, \ldots
$$

We do not consider here the question as to how the automorphisms of types (i), (ii), (iii) (and (iv)) might be supplemented to obtain a full generating set for Aut ( $F / V)$ (although in the case $F / V$ abelian, Theorem 2.2 below provides one answer).

\section{The Abelian Case}

Let $F$ be as above, free on the infinite set $\left\{x_{i} \mid i \in I\right\}$, and write $A=F /[F, F]$, the free abelianisation of $F$, and $A_{m}$ for $F / V$ where here $V$ is generated by $[F, F]$ together with all $m$ th powers of elements of $F(m>1)$; thus $A_{m}$ is the free abelian group of exponent $m$ and rank $|I|$. Our aim is to prove the abelian case of Theorem 1.1, namely:

Theorem 2.1. (Compare Macedońska-Nosalska [6].) Every bounded automorphism of $A$ or $A_{m}$ is induced by an automorphism of $F$ which is a finite product of GENTs of type (iv), that is, genetic.

(Note that in this, the abelian situation, the distinction between pre- and postmultiplication becomes immaterial.)

This theorem is a direct consequence of a result essentially due to Swan. To formulate this result we need the following concept. Let $\left\{y_{i} \mid i \in I\right\}$ be a free basis for $A$ (or $A_{m}$ ), and for $S \subseteq I$ denote by $\langle S\rangle$ the subgroup generated by the $y_{i}, i \in S$. We shall say that an automorphism $\theta$ of $A$ (or $A_{m}$ ) is $2 \times 2$ block-unitriangular relative to the given basis if there is a partition $I=I_{1} \amalg I_{2}$ such that $\left|I_{1}\right|=|I|=\left|I_{2}\right|$, and with respect to the direct decomposition

$$
A\left(\text { or } A_{m}\right)=\left\langle I_{1}\right\rangle \oplus\left\langle I_{2}\right\rangle,
$$


$\theta$ has the block matrix form

$$
\theta=\left(\begin{array}{ll}
1 & g \\
0 & 1
\end{array}\right)
$$

where the 1 's denote the identity maps on $\left\langle I_{1}\right\rangle$ and $\left\langle I_{2}\right\rangle$. (We are assuming the convention that the vectors on which $\theta$ acts are written as row-vectors to the left of the matrix (3).)

Theorem 2.2. (Swan; see [3, Section 2]) Every (bounded) automorphism $\varphi$ of $A$ (or $A_{m}$ ) is a product of $\leqslant 22$ (bounded) $2 \times 2$ block-unitriangular automorphisms all relative to a fixed basis $\left\{y_{i} \mid i \in I\right\}$.

Theorem 2.1 follows readily from this since an automorphism of the form (3) where the column-sums of the absolute values of the entries of the (infinite) matrix $g$ are bounded, by $N$ say, is the product of $\leqslant N$ GENTs of type (iv). (Note incidentally that in the more general situation where the columns of $g$ have bounded numbers of non-zero entries, $\theta$ is a finite product of automorphisms of the type mentioned at the beginning of Remark 3 above.)

Proof of Theorem 2.2: This largely follows Swan's proof in [3]. We give the proof for $A$ only, in the "bounded" situation. The proofs for $A_{m}$, and of the "unbounded" versions, differ insignificantly from this one.

(a) Consider first the case that our bounded automorphism $\varphi$ fixes all $y_{i_{1}}$ with $i_{1}$ in some subset $I_{1} \subseteq I$ of the same cardinality as $I$. Clearly, we may suppose that $I_{2}=I-I_{1}$ also has cardinality $|I|$, by reducing $I_{1}$ appropriately, if necessary. Then

$$
A=\left\langle I_{1}\right\rangle \oplus\left\langle I_{2}\right\rangle
$$

and relative to this decomposition we may write $\varphi$ in the block-matrix form

$$
\varphi=\left(\begin{array}{ll}
1 & 0 \\
h & k
\end{array}\right)
$$

with the understanding, as always, that elements of $A$ are to be written as row-vectors to the left of such matrices. Since $\varphi$ is bounded relative to the given basis, the maps $h$ and $k$, regarded as infinite matrices relative to the bases $\left\{y_{i_{1}} \mid i_{1} \in I_{1}\right\}$ and $\left\{y_{i_{2}} \mid i_{2} \in I_{2}\right\}$, are column-bounded in the sense, already noted, that the column-sums of the absolute values of their entries are bounded. Similarly, since

$$
\varphi^{-1}=\left(\begin{array}{cc}
1 & 0 \\
-k^{-1} h & k^{-1}
\end{array}\right),
$$


the same holds for $k^{-1}$ (and $k^{-1} h$ ). Since

$$
\varphi=\left(\begin{array}{ll}
1 & 0 \\
h & k
\end{array}\right)=\left(\begin{array}{ll}
1 & 0 \\
0 & k
\end{array}\right)\left(\begin{array}{cc}
1 & 0 \\
k^{-1} h & 1
\end{array}\right)
$$

and the last matrix in this equation has the form (3) and is bounded, it suffices to show that bounded automorphisms of the form

$$
\psi=\left(\begin{array}{ll}
1 & 0 \\
0 & k
\end{array}\right)
$$

are finite products of bounded automorphisms of the form (3).

For such automorphisms the result now follows by using "tricks of Whitehead and Eilenberg" ([3]): Partition $I_{1}$ into countably many subsets $E_{1}, E_{2}, \ldots$, each of cardinality $|I|$. Relative to the direct decomposition

$$
A=\left\langle I_{2}\right\rangle \oplus\left\langle E_{1}\right\rangle \oplus\left\langle E_{2}\right\rangle \oplus \ldots
$$

we may write the automorphism $\psi$ as

$$
k \oplus 1 \oplus 1 \oplus \ldots=\left(k \oplus k^{-1} \oplus k \oplus \ldots\right) \circ\left(1 \oplus k \oplus k^{-1} \oplus k \oplus \ldots\right)
$$

where the actions of the various $k$ 's on the corresponding $\left\langle E_{j}\right\rangle$ 's are similar to the action of $k$ on $\left\langle I_{2}\right\rangle$. The desired conclusion then follows from

$$
k \oplus k^{-1}=\left(\begin{array}{cc}
k & 0 \\
0 & k^{-1}
\end{array}\right)=\left(\begin{array}{cc}
1 & k-1 \\
0 & 1
\end{array}\right)\left(\begin{array}{ll}
1 & 0 \\
1 & 1
\end{array}\right)\left(\begin{array}{cc}
1 & k^{-1}-1 \\
0 & 1
\end{array}\right)\left(\begin{array}{cc}
1 & 0 \\
-k & 1
\end{array}\right) .
$$

(It follows that $\psi$ is expressible as a product of $\leqslant 8$ (bounded) automorphisms of the form (3), so that by (4) $\varphi$ is a product of $\leqslant 9$ such automorphisms.)

(b) Now let $\varphi$ be an arbitrary bounded automorphism of $A$ relative to the basis $\left\{y_{i} \mid i \in I\right\}$. Well-order $I$ so that it is order-isomorphic to the least ordinal of cardinality $|I|$. Analugously to [3, Section 2] we write $I$ as the union of an ascending chain

$$
\phi=I_{0} \subseteq J_{0} \subseteq I_{1} \subseteq J_{1} \subseteq \ldots
$$

of initial segments, defined by transfinite induction as follows: Assuming $I_{r-1}$ defined, choose $J_{r-1}$ to be the smallest initial segment of $I$ such that

$$
\left\langle I_{r-1}\right\rangle \cup\left(\left\langle I_{r-1}\right\rangle \varphi\right) \subseteq\left\langle J_{r-1}\right\rangle
$$

and consider

$$
\varphi:\left\langle I_{r-1}\right\rangle \oplus\left\langle I-I_{r-1}\right\rangle \rightarrow\left\langle J_{r-1}\right\rangle \oplus\left\langle I-J_{r-1}\right\rangle
$$


Let $i_{r}$ be the least element of $I-J_{r-1}$, and write

$$
\varphi^{-1}\left(y_{i_{r}}\right)=b_{r}+a_{r}, \text { where } b_{r} \in\left\langle I_{r-1}\right\rangle, a_{r} \in\left\langle I-I_{r-1}\right\rangle
$$

Then

$$
\varphi\left(a_{r}\right)=c_{r}+y_{i_{r}}, \text { where } c_{r}=\varphi\left(-b_{r}\right) \in\left\langle J_{r-1}\right\rangle,
$$

and since $c_{r}+y_{i_{r}}$ is a member of a free basis for $A$ which includes the $y_{j}$ with $j \in J_{r-1}$, it follows that $a_{r}$ is a meinber of a free basis for $A$ including the $y_{i}$ with $i \in I_{r-1}$. Writing $a_{r}=\sum \alpha_{t} y_{i_{t}}$, define $I_{r}$ to be the shortest initial segment of the well-ordered set $I$, containing $i_{r}$ (and therefore $J_{r-1}$ ), all those $i_{t}$ for which $\alpha_{t} \neq 0$, and the least element $k_{r}$ exceeding both $i_{r}$ and these $i_{t}$. Then $a_{r}$ is a member of a free basis for $\left\langle I_{r}-\left(I_{r-1} \cup\left\{k_{r}\right\}\right)\right\rangle$.

This defines $I_{r}$ when $r$ is not a limit ordinal. If $r$ is a limit ordinal, set $I_{r}=\bigcup_{j<r} I_{j}$.

Writing $H_{r}=\left\langle I_{r}-I_{r-1}\right\rangle$ for non-limit ordinals $r \geqslant 1$, we have $A=\bigoplus_{r} H_{r}$ where the sum is over all non-limit ordinals $0<r<|I|$, and where for each such $r$, the element $a_{r}$ defined as above is a member of a free basis for $\left\langle I_{r}-\left(I_{r-1} \cup\left\{k_{r}\right\}\right)\right\rangle=H_{r}^{\prime}$ say. Hence for each such $r$ we have $H_{r}=H_{r}^{\prime} \oplus\left\langle y_{k_{r}}\right\rangle$ with $a_{r}$ a free generator of $H_{r}^{\prime}$, so that there is an automorphism $\mu_{r}$ of $H_{r}$ of the form $\mu_{r}^{\prime} \oplus 1$ relative to this decomposition, satisfying $y_{i_{r}} \mu_{r}=y_{i_{r}} \mu_{r}^{\prime}=a_{r}$ (and $y_{k_{r}} \mu_{r}=y_{k_{r}}$ ). Since $\sum\left|\alpha_{t}\right|$ is bounded (over all $r$ ) the $\mu_{r}$ may moreover be chosen so that the autornorphism $\mu=\bigoplus_{r} \mu_{r}$ is bounded. Thus with respect to the direct decomposition $A=\langle I-K\rangle \oplus\langle K\rangle$, where $K$ is the set of all $k_{r}$, the automorplism $\mu$ of $A$ has the form $\mu^{\prime} \oplus 1$, and so by the conclusion of part (a) is a product of $\leqslant 4$ bounded automorphisms of the form (3).

Consider next the map $\nu$ given by

$$
y_{i} \nu= \begin{cases}y_{i} & \text { for } i \neq \text { any } i_{r}, \\ c_{r}+y_{i_{r}} & \text { for } i=i_{r} .\end{cases}
$$

Since $c_{r} \in\left\langle J_{r-1}\right\rangle$, while $i_{r}>j$ for all $j \in J_{r-1}$, this defines an automorphism $\nu$ of $A$, bounded in view of (5) and (6). Since $\nu$ fixes the $y_{k_{r}}$, it fixes $|I|$ elements of the basis $\left\{y_{i} \mid i \in I\right\}$, and so by part (a) of the proof $\nu$ is a product of $\leqslant 9$ bounded $2 \times 2$ block-unitriangular automorphisms relative to that basis. Since $y_{i_{r}} \mu \varphi \nu^{-1}=y_{i_{r}}$, the same is true of $\mu \varphi \nu^{-1}$. Hence $\varphi$ is a product of $\leqslant 9+9+4=22$ such automorphisms, as claimed.

\section{The NILPotent CASE}

IIaving disposed of the case $c=1$ of Theorem 1.1, we proceed to the proof of the [ull result, using induction on $c$, and basing our argument essentially on Section 3 of [1]. 
Thus suppose $c>1$ and that the conclusion of Theorem 1.1 holds for smaller classes. Let $\theta^{\prime}$ be the automorphism induced by $\theta$ on $F / \gamma_{c}(F) V$. By the inductive hypothesis $\theta^{\prime}$ can be lifted to a genetic automorphism $\mu_{1}$ of $F$. Denoting by $\theta_{1}$ the automorphism of $F / V$ induced by $\mu_{1}$, we consider the automorphism $\theta_{2}=\theta \theta_{1}^{-1}$ of $F / V$. Since $\mu_{1}$ is certainly (freely) bounded, $\theta_{1}$ is bounded, and therefore so is $\theta_{2}$. (It is easy to show, by induction on the class, that the product of two bounded automorphisms is again bounded.) Since $\theta_{1}$ lifts to a genelic automorphism of $F$, it suffices to show that $\theta_{2}$ can be so lifted. Since $\theta_{2}$ induces the identity map on $F / \gamma_{c}(F) V$, the image under $\theta_{2}$ of each free generator $x_{i} V$ of $F / V$ may be pul in the form

$$
\left(x_{i} V\right) \theta_{2}=\left(x_{i} c_{i, 1} c_{i, 2} \ldots c_{i, N}\right) V
$$

where each $c_{i, \lambda}, \lambda=1,2, \ldots, N$, is either trivial or a left-normed commutator of weight $c$ in the $x_{j}, j \in I$, and $N$ is the bound on $\theta_{2}$. Since $\theta_{2}=\theta_{2}^{(1)} \theta_{2}^{(2)} \ldots \theta_{2}^{(N)}$, where $\theta_{2}^{(\lambda)}$ is defined by

$$
\left(x_{i} V\right) \theta_{2}^{(\lambda)}=\left(x_{i} c_{i, \lambda}\right) V, i \in I,
$$

it suffices to consider automorphisins $\varphi$ of $F / V$ of the form

$$
\left(x_{i} V\right) \varphi=\left(x_{i}\left[x_{i_{1}}, x_{i_{2}}, \ldots, x_{i_{c}}\right]\right) V, i \in I
$$

Before proceeding further with the general inductive step, we need to consider separately, as in [1, Section 3], the case $c=2$. By the above we may in this case suppose

$$
\left(x_{i} V\right) \varphi=\left(x_{i}\left[x_{i_{1}}, x_{i_{2}}\right]\right) V, i \in I
$$

where now we are assuming $V \geqslant \gamma_{3}(F)$.

Consider any fixed $i$ in (9) for which $i_{1} \neq i_{2}$. If $i_{1}=i$, then $x_{i}\left[x_{i_{1}}, x_{i_{2}}\right]=$ $x_{i}\left[x_{i}, x_{i_{2}}\right]=x_{i_{2}}^{-1} x_{i} x_{i_{2}}$, while if $i_{2}=i$, then

$$
x_{i}\left[x_{i_{1}}, x_{i_{2}}\right] V=x_{i}\left[x_{i_{1}}, x_{i}\right] V=x_{i}\left[x_{i}, x_{i_{1}}^{-1}\right] V=\left(x_{i_{1}} x_{i} x_{i_{1}}^{-1}\right) V
$$

Hence if either $i_{1}=i$ or $i_{2}=i$, then the automorphism $\eta_{1}$ of $F / V$ induced by the automorphism $\eta$ of $F$ conjugating $x_{i}$ by $x_{i_{2}}^{-1}$ or by $x_{i_{1}}$, as the case may be, and leaving fixed all $x_{j}, j \neq i$, satisfies:

$$
\left(x_{i} V\right) \varphi \eta_{1}=x_{i} V ; \quad\left(x_{j} V\right) \varphi \eta_{1}=\left(x_{j} V\right) \varphi, j \neq i
$$

If $i_{1} \neq i \neq i_{2}$, let $\eta$ be the automorphism of $F$ sending $x_{i}$ to $x_{i}\left[x_{i_{1}}, x_{i_{2}}\right]^{-1}$ and fixing all $x_{j}, j \neq i$. The automorphism $\eta_{1}$ of $F / V$ induced by this $\eta$ will again satisly (10). 
Since in every case $\eta$ is a product of $\leqslant 8$ elementary Nielsen transformations of $F$, we see that individual $\left(x_{i} V\right) \varphi$ of the form (9) may be transformed to $x_{i} V$ by applying a free automorphism which is a product of $\leqslant 8$ elementary Nielsen transformations.

The question remains as to how such transformations can be effected simultaneously for all $\left(x_{i} V\right) \varphi$ by means of a single genelic aulomorphism of $F$. To answer this question we reformulate it in terms of graph theory. Consider the directed graph $\Gamma$ whose vertices are just the elements $\left(x_{i} V\right) \varphi, i \in I$, and whose directed edges are defined as follows: For $v_{i}=\left(x_{i} V\right) \varphi$ as in (9) where $i_{1} \neq i_{2}$, introduce an edge leading from $v_{i}$ to $v_{i_{1}}$ if $i \neq i_{1}$, and also from $v_{i}$ to $v_{i_{2}}$ if $i \neq i_{2}$. Now in transforming $\left(x_{i} V\right) \varphi$ to $x_{i} V$ in the manner described above, one execules a succession of (at most 4) pre- and/or post-multiplications of $v_{i}=\left(x_{i} V\right) \varphi$ by $v_{i_{1}}^{ \pm 1}$ and/or $v_{i_{2}}^{ \pm 1}$ (both if $i_{1} \neq i \neq i_{2}$ ). (This is the effect of following $\varphi$ by $\eta_{1}$ in (10).) We encode this operation in $\Gamma$ by colouring the $\leqslant 2$ edges beginning at $v_{i}$ with a single colour. Now in using $v_{i_{1}}$ and/or $v_{i_{2}}$ to reduce $v_{i}$ we are prohibited from simultaneously carrying out an analogous reduction of $v_{i_{1}}$ and/or $v_{i_{2}}$, but this is the only restriction on carrying out a set of such reducing operations simultaneously. In terms of $\Gamma$ this translates into the following criterion: $A$ partial edge-colouring of $\Gamma$ by a single colour (say red) corresponds to the application of an automorphism of $F / V$ induced by a genetic automorphism of $F$ which on certain $v_{i}$ acts like $\eta_{1}$ (see (10)), precisely if the $\leqslant 2$ edges out of these $v_{i}$ are coloured red, and no others, and no directed path of length 2 is coloured red. It follows that all $\left(x_{i} V\right) \varphi \neq x_{i} V$ can be reduced by means of a finite product of such genetic automorphisms if and only if $\Gamma$ can be edge-coloured with finitely many coluurs in such a way that the $\leqslant 2$ edges beginning at each vertex receive the same colour, and no directed path of length 2 is monochromatic.

That $\Gamma$ can be so coloured is the case $c=2$ of the following simple combinatorial leinma, whose proof we relegate to the end of the paper.

LEMmA 3.1. Let $\Gamma$ be a (possibly infinite) directed graph without loops each of whose vertices is the initial vertex of $\leqslant c$ edges. Then the edges of $\Gamma$ can be coloured with $\leqslant 3 c$ colours in such a way that no directed path of length 2 is monochromatic, and furthermore so that all edges with a common initial vertex are coloured with the same colour.

We now sketch the inductive step in the proof of Theorem 1.1 , from $c-1(\geqslant 2)$ to $c$. We shall need the following lemma (valid for all $c \geqslant 2$ ).

LEMma 3.2. Let $F$, free on $\left\{x_{i} \mid i \in I\right\}, I$ infinite, and $V \geqslant \gamma_{c+1}(F)$ be as before, and let $p, p_{1}, \ldots, p_{c}$ be (not necessarily distinct) eleınents of $I$. The automorphism $\pi$ of $F / V$ defined by:

$$
\left(x_{p} V\right) \pi=\left(x_{p}\left[x_{p_{1}}, x_{p_{2}}, \ldots, x_{p_{c}}\right]\right) V ; \quad\left(x_{i} V\right) \pi=x_{i} V, i \neq p
$$


can be lifted to an automorphism of $F$ which is a product of $<8^{c}$ GENTs of type (iv) each of which fixes those of $x_{p_{1}}, \ldots, x_{p_{c}}$ different from $x_{p}$.

Remark. Note for later use that, assuming this lemma true, then given any infinite subset $S \subset I$ containing $p, p_{1}, \ldots, p_{c}, \pi$ may in fact be lifted to an automorphism of $F$ of the form $f * 1$ relative to the free decomposition $F=\langle S\rangle *\langle I-S\rangle$, where $f$ is an automorphism of the free group $\langle S\rangle$ which is a finite product of GENTs of type (iv) as in the lemina.

Proof of Lemma 3.1: We adapt the basic idea of [1, Section 3]. The case $c=2$ having been deall with above, we suppose $c>2$ and that the statement of the lemma is true with $c-1$ in place of $c$. We may assume also that $p_{1} \neq p_{2}$ since otherwise the lemma is trivial. We define a sequence of elemenls $q_{1}, q_{2}, \ldots$ of $I$, and a sequence of automorphisms $\pi_{1}, \xi_{1}, \pi_{2}, \xi_{2}, \ldots$ of $F$, as follows: Partition $I-\left\{p, p_{1}, \ldots, p_{c}\right\}$ into countably many infinite subsets $Q_{1}, Q_{2}, \ldots$, write $P_{1}=Q_{1} \cup\left\{p, p_{1}, \ldots, p_{c}\right\}$, and choose $q_{1} \in Q_{1}$. By the inductive hypothesis the automorphism of $F / \gamma_{c}(F) V$ defined by:

$$
x_{p}\left(\gamma_{c}(F) V\right) \mapsto x_{p}\left[x_{q_{1}}, x_{p_{3}}, \ldots, x_{p_{c}}\right] \gamma_{c}(F) V ; \quad x_{i}\left(\gamma_{c}(F) V\right) \text { fixed for } i \neq p,
$$

can be lifted to an automorphism $\pi_{1}$ of $F$ of the form $\pi_{1}^{\prime} * 1$ relative to the free decomposition $F=\left\langle P_{1}\right\rangle *\left\langle I-P_{1}\right\rangle$ (see the above remark) such that $\pi_{1}^{\prime}$ is a product of $<8^{c-1}$ GENTs of type (iv) each fixing those of $x_{p_{1}}, \ldots, x_{p_{c}}$ different from $x_{p}$. Define $\xi_{1}$ by:

$$
x_{q_{1}} \xi_{1}=x_{q_{1}}\left[x_{p_{1}}, x_{p_{2}}\right] ; \quad x_{i} \xi=x_{i}, i \neq q_{1} .
$$

A direct calculation shows that, working now modulo $V$, the automorphism $\pi_{1} \xi_{1} \pi_{1}^{-1} \xi_{1}^{-1}=\left[\pi_{1}^{-1}, \xi_{1}^{-1}\right]$ induces the automorphism of $F / V$ given by:

$$
\begin{aligned}
& x_{p} V \mapsto x_{p}\left[x_{p_{1}}, x_{p_{2}}, \ldots, x_{p_{c}}\right] V ; \\
& x_{q_{1}} V \mapsto \begin{cases}x_{q_{1}} V, & \text { if } p_{1} \neq p \neq p_{2}, \\
x_{q_{1}}\left[x_{p_{3}}, x_{q_{1}}, x_{p_{4}}, \ldots, x_{p_{c}}, x_{p_{2}}\right] V, & \text { if } p_{1}=p, \\
x_{q_{1}}\left[x_{q_{1}}, x_{p_{3}}, x_{p_{4}}, \ldots, x_{p_{c}}, x_{p_{1}}\right] V, & \text { if } p_{2}=p ;\end{cases} \\
& x_{i} V \mapsto x_{i} V, \quad \text { if } i \neq p, q_{1} .
\end{aligned}
$$

If $p_{1} \neq p \neq p_{2}$, then $\left[\pi_{1}^{-1}, \xi_{1}^{-1}\right]$ induces $\pi$ and we have the desired conclusion. Otherwise define $P_{2}=\left(Q_{2} \cup\left\{q_{1}, p_{1}, \ldots, p_{c}\right\}\right)-\{p\}$, choose $q_{2} \in Q_{2}$, and let $\pi_{2}$ be an automorphism of $F$ (guaranteed by the inductive hypothesis) of the form $\pi_{2}^{\prime} * 1$ relative to the free decomposition $\left\langle P_{2}\right\rangle *\left\langle I-P_{2}\right\rangle$ such that $\pi_{2}^{\prime}$ is a product of $<8^{c-1}$ GENTs of type (iv) each fixing those of $x_{p_{1}}, \ldots, x_{p_{c}}$ different from $x_{p}$, which induces 
the automorphism of $F / \gamma_{c}(F) V$ given by (compare (11)):

$$
\begin{aligned}
& x_{q_{1}}\left(\gamma_{c}(F) V\right) \mapsto \begin{cases}x_{q_{1}}\left[x_{q_{2}}, x_{p_{4}}, \ldots, x_{p_{c}}, x_{p_{2}}\right]^{-1} \gamma_{c}(F) V, & \text { if } p_{1}=p \\
x_{q_{1}}\left[x_{q_{2}}, x_{p_{4}}, \ldots, x_{p_{c}}, x_{p_{1}}\right]^{-1} \gamma_{c}(F) V, & \text { if } p_{2}=p ;\end{cases} \\
& x_{i}\left(\gamma_{c}(F) V\right) \mapsto x_{i}\left(\gamma_{c}(F) V\right) \quad \text { for } i \neq q_{1} .
\end{aligned}
$$

Define $\xi_{2}$ by (compare (12)):

$$
\begin{aligned}
& x_{q_{2}} \xi_{2}= \begin{cases}x_{q_{2}}\left[x_{p_{3}}, x_{q_{1}}\right], & \text { if } p_{1}=p \\
x_{q_{2}}\left[x_{q_{1}}, x_{p_{3}}\right], & \text { if } p_{2}=p\end{cases} \\
& x_{i} \xi_{2}=x_{i} \quad \text { for } i \neq q_{2} .
\end{aligned}
$$

Then the free automorphism $\left[\pi_{2}^{-1}, \xi_{2}^{-1}\right]$ induces the automorphism of $F / V$ defined by (compare (13)):

$$
\begin{aligned}
& x_{q_{1}} V \mapsto \begin{cases}x_{q_{1}}\left[x_{p_{3}}, x_{q_{1}}, x_{p_{4}}, \ldots, x_{p_{c}}, x_{p_{2}}\right]^{-1} V, & \text { if } p_{1}=p, \\
x_{q_{1}}\left[x_{q_{1}}, x_{p_{3}}, x_{p_{4}}, \ldots, x_{p_{c}}, x_{p_{1}}\right]^{-1} V, & \text { if } p_{2}=p ;\end{cases} \\
& x_{q_{2}} V \mapsto \begin{cases}x_{q_{2}}\left[x_{q_{2}}, x_{p_{4}}, \ldots, x_{p_{c}}, x_{p_{2}}, x_{p_{3}}\right]^{-1} V & \text { if } p_{1}=p, \\
x_{q_{2}}\left[x_{q_{2}}, x_{p_{4}}, \ldots, x_{p_{c}}, x_{p_{1}}, x_{p_{3}}\right] V, & \text { if } p_{2}=p ;\end{cases} \\
& x_{i} V \mapsto x_{i} V \quad \text { for } i \neq q_{1}, q_{2} .
\end{aligned}
$$

From this and (13) we see that the product $\left[\pi_{1}^{-1}, \xi_{1}^{-1}\right]\left[\pi_{2}^{-1}, \xi_{2}^{-1}\right]$ induces the automorphism of $F / V$ defined by (compare (13)):

$$
\begin{aligned}
& x_{p} V \mapsto x_{p}\left[x_{p_{1}}, x_{p_{2}}, \ldots, x_{p_{c}}\right] V ; \\
& x_{q_{2}} V \mapsto \begin{cases}x_{q_{2}}\left[x_{q_{2}}, x_{p_{4}}, \ldots, x_{p_{c}}, x_{p_{2}}, x_{p_{3}}\right]^{-1} V, & \text { if } p_{1}=p, \\
x_{q_{2}}\left[x_{q_{2}}, x_{p_{4}}, \ldots, x_{p_{c}}, x_{p_{1}}, x_{p_{3}}\right] V, & \text { if } p_{2}=p ;\end{cases} \\
& x_{i} V \mapsto x_{i} V \quad \text { for } i \neq p, q_{2} .
\end{aligned}
$$

One continues (inductively) in this way, choosing $q_{n} \in Q_{n}$, writing $P_{n}=$ $\left(Q_{n} \cup\left\{q_{n-1}, p_{1}, \ldots, p_{c}\right\}\right)-\{p\}$, and using the inductive hypothesis to obtain $\pi_{n}$, in the form $\pi_{n}^{\prime} * 1$ relative to the free decomposition $F=\left\langle P_{n}\right\rangle *\left\langle I-P_{n}\right\rangle$, as a product of $<8^{c-1}$ GENT's of type (iv) each involving only the $x_{i}$ with $i \in P_{n}$ and fixing those of $x_{p_{1}}, \ldots, x_{p_{c}}$ different from $x_{p}$, and inducing an automorphism of $F / \gamma_{c}(F) V$ moving only $x_{q_{n-1}}\left(\gamma_{c}(F) V\right.$ ) (analogously to (14)). The automorphism $\xi_{n}$ is defined, analogously to (15), to move only $x_{q_{n}}$, by post-multiplying it by a commutator $\left[x_{i_{n}}, x_{j_{n}}\right]$ where one of $i_{n}, j_{n}$ is $q_{n-1}$ and the other is one of $p_{1}, \ldots, p_{c}$. Since $q_{n} \notin\left\{p_{1}, \ldots, p_{c}\right\}$ and the subsets of $\left\{x_{i} \mid i \in I\right\}$ involved in the GEN'T's composing the automorphisms 
$\pi_{n}, \pi_{n+k}, k \geqslant 2$, intersect in a subset of $\left\{p_{1}, \ldots, p_{c}\right\}-\{p\}$ (contained as they are in $\left.P_{n}, P_{n+k}\right)$, the automorphisms $\pi_{1}, \xi_{1}, \pi_{2}, \xi_{2}, \ldots$ ultimately obtained have the following properties:

( $\alpha$ ) the automorphism $\pi$ is induced by the infinite product $\prod_{n=1}^{\infty}\left[\pi_{n}^{-1}, \xi_{n}^{-1}\right]$ (which makes sense since each $x_{i}$ is fixed by almost every $\pi_{n}, \xi_{n}$ );

$(\beta)$ the commutators $\left[\pi_{n}^{-1}, \xi_{n}^{-1}\right]$ induce pairwise commuting automorphisms of $F / V$;

( $\gamma$ ) for every $n=1,2, \ldots$, and every $k \geqslant 2$, the automorphisms $\pi_{n}, \xi_{n}$ both commute with $\pi_{n+k}, \xi_{n+k}$;

$(\delta)$ each of the infinite products

$$
\pi_{1}^{ \pm 1} \pi_{3}^{ \pm 1} \pi_{5}^{ \pm 1} \ldots, \quad \pi_{2}^{ \pm 1} \pi_{4}^{ \pm 1} \pi_{6}^{ \pm 1} \ldots
$$

is expressible as a product of $<8^{c-1}$ GEN'Ss of type (iv), and each of

$$
\xi_{1}^{ \pm 1} \xi_{3}^{ \pm 1} \xi_{5}^{ \pm 1} \ldots, \quad \xi_{2}^{ \pm 1} \xi_{4}^{ \pm 1} \xi_{8}^{ \pm 1} \ldots
$$

is expressible as a product of $\leqslant 4$ GENTs of type (iv).

Writing $\widehat{\pi}_{n}, \widehat{\xi}_{n}$ for the induced automorphisms of $F / V$, we obtain from $(\alpha),(\beta)$ and $(\gamma)$ that

$$
\pi=\left[\widehat{\pi}_{1}^{-1} \widehat{\pi}_{3}^{-1} \widehat{\pi}_{5}^{-1} \ldots, \widehat{\xi}_{1}^{-1} \hat{\xi}_{3}^{-1} \widehat{\xi}_{5}^{-1} \ldots\right] \circ\left[\widehat{\pi}_{2}^{-1} \widehat{\pi}_{4}^{-1} \widehat{\pi}_{6}^{-1} \ldots, \widehat{\xi}_{2}^{-1} \widehat{\xi}_{4}^{-1} \hat{\xi}_{6}^{-1} \ldots\right],
$$

and it then follows from $(\delta)$ that $\pi$ is induced by a product of $<4\left(8^{c-1}+4\right)<8^{c}$ GENTs of type (iv), completing the proof of the lemma.

We are now in a position to complete the proof of Theorem 1.1 in the case $c>2$. We have already observed that by induction it suffices to consider automorphisms $\varphi$ of $F / V$ of the form (8):

$$
\left(x_{i} V\right) \varphi=x_{i}\left[x_{i_{1}}, x_{i_{2}}, \ldots, x_{i_{c}}\right] V, i \in I .
$$

Let $I=I_{1} \amalg I_{2}$ where $\left|I_{1}\right|=|I|=\left|I_{2}\right|$, and define $\varphi_{1}$ to agree with $\varphi$ on $\left\langle I_{1}\right\rangle$ and fix the elements of $\left\langle I_{2}\right\rangle$, and vice versa for $\varphi_{2}$. Then $\varphi=\varphi_{1} \varphi_{2}$, so that it suffices to show that $\varphi_{1}$ is genetic.

Let $J$ denote the subset of indices $j \in I$ for which $\left(x_{j} V\right) \varphi_{1} \neq x_{j} V$. Partition $I_{2}$ into $|J|$ infinite subsets $A_{j}, j \in J$, and write $B_{j}=A_{j} \cup\left\{j, j_{1}, j_{2}, \ldots, j_{c}\right\}$. For each $j \in J$ consider the automorphism $\varphi_{j}$ of $F / V$ agreeing with $\varphi_{1}$ on $x_{j} V$ and fixing all $x_{i} V$ with $i \neq j$ :

$$
\left(x_{j} V\right) \varphi_{j}=x_{j}\left[x_{j_{1}}, x_{j_{2}}, \ldots, x_{j_{c}}\right] V ; \quad\left(x_{i} V\right) \varphi_{j}=x_{i} V \text { for } i \neq j .
$$


Then by Lemma 3.2 (and the remark following it) each $\varphi_{j}$ is induced by an automorphism $f_{j}$ of $F$ of the form $f_{j}^{\prime} * 1$ relative to the free decomposition $F=\left\langle B_{j}\right\rangle *\left\langle I-B_{j}\right\rangle$, where $f_{j}^{\prime}$ is expressible as a product of $<8^{c}$ GENTs of type (iv) each fixing those of $x_{j_{1}}, \ldots, x_{j_{c}}$ different from $x_{j}$.

We now introduce a directed graph $\Gamma_{1}$ analogous to that exploited in the case $c=2$. The vertices $v_{i}$ of $\Gamma_{1}$ are the $\left(x_{i} V\right) \varphi, i \in I$, and each $v_{j}, j \in J$, is joined by an edge directed to every $v_{i}$ with $i \in B_{j}-\{j\}$. Much as before we encode the automorphism $\varphi_{j}$ in $\Gamma_{1}$ as a colouring of all the edges emanating from $v_{j}$ with the same colour (red, say); a partial edge-colouring of $\Gamma_{1}$ with the colour red then corresponds to a genetic automorphism executing various of the $\varphi_{j}$ simultaneously, if all edges out of each vertex are red (or else none is coloured) and no directed path of length 2 is red. Hence $\varphi_{1}$ is genetic if $\Gamma_{1}$ can be coloured with finitely many colours so that all edges out of each vertex receive the same colour and no directed path of length 2 is monochromatic. Now each vertex $v_{\text {, with }} s \in I_{2}$, has no edges leading out of it (since $x_{s} V$ is fixed by $\varphi_{1}$ ) and precisely one edge terminating at it (namely that from $v_{j}$ to $v_{s}$ if $s \in A_{j}$ ); call this edge $e_{s}$. If an edge-colouring of $\Gamma_{1}$ is such that all edges out of each verlex receive the same colour and there is a monochromatic (red, say) directed path of length 2 of which $e_{e}$ is one of the edges, then $v_{j}$ must be the midpoint of that path. Denote by $e$ the first edge of that directed path. Since $\varphi_{1}$ does not fix $x_{j} V$ there is another edge $e^{\prime}$ out of $v_{j}$ (terminating in one of $v_{j_{1}}, \ldots, v_{j_{c}}$ ), and by the condition on our edge-colouring this edge will also be red. Hence the pair $e, e^{\prime}$ makes up a directed red path of lengih 2 no vertex of which is a $v_{s}$ with $s \in I_{2}$. The upshot is that in deciding whether or not an edge-colouring of $\Gamma_{1}$ of the desired sort is possible we may delete all vertices $v_{s}$ with $s \in I_{2}$, together with the edges terminating at them. The resulting graph $I$ then has $\leqslant c$ edges emanating from each vertex, and Lemma 3.1 now applies.

\section{Proof of Lemma 3.1}

We first show, by induction on $c$, that $\Gamma$ can be coloured with $\leqslant 3 c$ colours in such a way that no directed path of length 2 is monochromatic. In the case $c=1$ it is easy to see that each connected component of $\Gamma$ is either a tree directed downwards towards its root, or else consists of a single directed closed path without repeated edges, possibly with "downwards - directed" trees atlached to it by their roots. It is not difficult to see that such a graph can be coloured in the desired way using $\leqslant 3$ colours.

Suppose now that $c>1$. For eacli vertex $v$ with $>0$ edges leading out of it, choose exactly one of these edges $e_{v}$, and let $\Delta$ denote the (directed) subgraph consisting of all the edges $e_{v}$ together with their initial and terminal vertices. Then by the case $c=1$, $\triangle$ can be coloured appropriately with $\leqslant 3$ colours, and by the inductive hypothesis 
the complementary subgraph $\Omega$ consisting of the edges outside $\Delta$ together with their initial and terminal vertices, can be coloured with $\leqslant 3(c-1)$ colours appropriately. Hence $\Gamma$ itself can be coloured in the desired way using $\leqslant 3(c-1)+3=3 c$ colours.

It remains to show that there is such an edge-colouring of $\Gamma$ with the additional property that edges with a common initial vertex have the same colour. Perform the following operation on $\Gamma$ : for each vertex $v$, identify with a single directed edge all edges emanating from $v$ (and identify their terminal vertices with a single vertex), doing this simultaneously for all vertices. The resulting directed graph $\widehat{\Gamma}$ again has the property that each vertex is initial for $\leqslant c$ edges, so that, by the first part of the proof, $\widehat{\Gamma}$ can be edge-coloured with $\leqslant 3 c$ colours with no directed path of length 2 monochromatic. If we now coluur the edges of the original graph $\Gamma$ so that the natural identification map $E(\Gamma) \rightarrow E(\widehat{\Gamma})$ between the edge-scts of $\Gamma$ and $\widehat{\Gamma}$ is colour-preserving, then this edge-colouring has both desired properties.

\section{REFERENCES}

[1] R.M. Bryant and $O$. Macedońska, 'Autoniorphisms of relatively free nilpotent groups of infinite rank', J. Algebra (to appear).

[2] D.F. Cohen, 'Characteristic subgroups of some relatively free groups', J. London Math. Soc. 43 (1968), 445-451.

[3] J.M. Cohen, 'Aspherical 2-complexes', J. Pure Appl. Algebra 12 (1978), 101-110.

[4] R. Cohen, 'Classes of automorphisms of free groups of infinite rank', Trans. Amer. Math. Soc. 177 (1973), 99-120.

[6] M. Hall Jr., The Theory of Groups (Macnillan, New York, 1959).

[0] O. Macedońska-Nosalska, 'The abelian case of Solitar's conjecture on infinite Nielsen transformations', Canad. Math. Bull. 28 (1985), 223-230.

[7] W. Magnus, A. Karrass and D. Solitar, Combinatorial Group Theory (Interscience, New York, $1966)$.

Department of Mathematics

York University

Downsview, Toronto

Ontario

Canada 